\title{
Proton beam therapy for renal pelvis and ureter cancer: A report of 5 cases and a literature review
}

\author{
TAKASHI IIZUMI, HITOSHI ISHIKAWA, YUTA SEKINO, KAYOKO OHNISHI, \\ MASASHI MIZUMOTO, TETSUO NONAKA and HIDEYUKI SAKURAI \\ Department of Radiation Oncology and Proton Medical Research Center, \\ University of Tsukuba, Faculty of Medicine, Tsukuba, Ibaraki 305-8576, Japan
}

Received September 24, 2018; Accepted April 17, 2019

DOI: $10.3892 /$ mco.2019.1861

\begin{abstract}
Standard treatment for localized renal pelvis and ureter cancer is surgery. Previously, the primary role of radiation therapy (RT) in cancer treatment was to control pain and hemostasis as palliative or as adjuvant therapy following surgery. In this report, we describe 5 patients with the disease treated with proton beam therapy (PBT) as curative treatment. Between September 2009 and July 2013, 5 males with renal pelvis $(n=3)$ or ureter $(n=2)$ cancer were treated by PBT with hypofractionated [72.6 Gy relative biological effectiveness (RBE)/22 fractions] or conventional [64-66 Gy (RBE)/32-33 fractions] fractionation. The median patient age was 72 years (range, 59-85 years). Three patients were deemed unfit for surgery. Local hypofractionated PBT was performed in 2 patients with T1-2N0M0 diseases, while prophylactic lymph node irradiation using a patch irradiation technique was performed for the remaining 3 patients, who had T3-4 disease. Two patients with T3-4 disease received chemotherapy prior to definitive PBT. No serious acute or late toxicities were observed in any patient. Local tumor control was achieved in 3 patients $(60 \%)$; however, distant metastases were observed in 2 patients. Four of the five patients $(80 \%)$ evaluated in the present study survived for $>3$ years. The data is limited; however, PBT appears to be a potential option for patients with renal pelvis or ureter cancer, especially for those who are unsuitable for radical surgery.
\end{abstract}

\section{Introduction}

While urothelial carcinoma is a very common tumor type, involvement of the upper urinary tract is relatively uncommon,

Correspondence to: Dr Hitoshi Ishikawa, Department of Radiation Oncology and Proton Medical Research Center, University of Tsukuba, Faculty of Medicine, 2-1-1 Amakubo, Tsukuba, Ibaraki 305-8576, Japan

E-mail: hishikawa@pmrc.tsukuba.ac.jp

Key words: proton therapy, renal pelvis cancer, ureter cancer, radiation therapy, survival, morbidity accounting for 5 to $10 \%$ of all primary urothelial carcinomas (1-3). The gold standard for curative treatment of localized renal pelvis and ureter cancer is open or laparoscopic surgery (4). Due to a lack of data about the disease, the exact role of nonsurgical therapies, including radiation therapy (RT), remains unclear; the primary role of RT has been considered to be in controlling pain and hemorrhage as palliation or preventing recurrence after surgery as adjuvant therapy $(5,6)$. However, delivery of curative doses safely is challenging, as the small intestine and colon may be extensively irradiated during photon RT for patients with these tumors. Ding et al (7), investigated outcomes of 1,910 patients with primary transitional cell carcinoma of the ureter using the Surveillance, Epidemiology, and End Results database, and the 5-year overall survival rates of surgery only and RT only for stage I-II patients were 59.6 and $0 \%$, respectively. The patient's backgrounds of the two groups were unadjusted, but RT exerted a very limited impact on clinical outcomes as a curative treatment. Therefore, it is necessary for patients unfit for surgery including patients with unresectable tumors or medically inoperable condition to devise new approaches in the curative setting.

Protons have unique physical characteristics called Bragg peaks, and a region of uniform dose can be fit to the location and size of a tumor by overlaying several peaks, known as a spread-out Bragg peak (SOBP) $(8,9)$. Thus, proton beam therapy (PBT) can deliver conformal high-dose irradiation to the target while minimizing radiation-induced complications in surrounding healthy tissue. In recent decades, the efficacy and feasibility of PBT for urological malignancies including bladder cancer and prostate cancer have been reported with an increase of PBT facilities in clinical operation worldwide (10-14). On the other hand, no reports regarding PBT for renal pelvis and ureter cancer have been published. In our institute, we have treated 5 patients treated with definitive PBT, and herein describe our experience to explore the potential effectiveness of PBT as a curative treatment for renal pelvis and ureter cancer patients unfit for surgery.

\section{Patients and methods}

Between September 2009 and July 2013, 5 patients with renal pelvis or ureter cancer were definitively treated with PBT at our hospital. Before PBT, a cystoscopy was performed for 
all patients and confirmed no evidence of malignancy in the bladder and urethra. Patient characteristics are summarized in Table I. The median age of was 72 years (range, 59 to 85 years). The initial Eastern Cooperative Oncology Group performance status was 0 or 1 , but 3 patients were unfit for surgery (medically inoperable, $\mathrm{n}=1$; unresectable, $\mathrm{n}=2$ ). Primary tumor sites included the renal pelvis $(\mathrm{n}=3)$ and ureter $(\mathrm{n}=2)$. In reference to previous reports for RT for cancer of the upper urinary tract, clinical staging but not pathological staging was used in the present study $(15,16)$. Two patients with stage IV (case 3 and case 5) received systemic chemotherapy and the response to the chemotherapy was so good that these patients were sent to our hospital to obtain a control of the localized residual tumors.

Proton beam therapy. PBT for all 5 patients was performed without combining it with photon RT. Treatment planning for PBT involved respiratory-synchronized computed tomography $(\mathrm{CT})$ at $5-\mathrm{mm}$ intervals in the treatment position during the expiratory phase, and the images were transferred directly to a treatment planning system (Hitachi Co., Ltd., Japan). Proton beams were delivered in double-scattering mode during the expiratory phase using an end-expiratory gated system controlled by a laser range-finder that monitors the movement of the patient's body surface caused by respiratory motion (17). The beams were synchronized with respiration, and the position was examined by fluoroscopy during each treatment session. Clinical target volume (CTV), which was defined as a macroscopic tumor volume that included visible tumors with 5 to $10-\mathrm{mm}$ margins, was covered by $>95 \%$ of the prescribed dose at the isocenter by selection of appropriate ports and margins. The relative biological effective (RBE) value for protons was set to be 1.1 in our institute, and the irradiation dose was expressed in Gy (RBE) [physical proton dose (Gy)xRBE].

The first 2 cases with T1-2N0M0 disease were treated with local PBT at a total dose of 66.0/72.6 Gy (RBE) in 22 fractions with a fractional dose of 3.0/3.3 Gy (RBE). Since a designed seamless irradiation technique called the 'patch-fields technique' for extended whole mediastinal PBT for esophageal cancer was developed in $2010(18,19)$, our treatment policy for PBT for renal pelvis and ureter cancer has changed. Namely, total doses of 60/66 Gy (RBE) in 33 fractions with conventional fractionation were administered with prophylactic lymph node irradiation, including the bilateral paraaortic lymph nodes area and ipsilateral common iliac nodes, to the entire ureter and through the renal pelvis to the ureteral orifice using extended PBT fields. After 36.4/40 Gy (RBE) of irradiation was administered, shrunken PBT fields covering gross tumor volumes were used for an additional boost to 60/66 Gy (RBE).

\section{Results}

This retrospective analysis was approved by the ethical committee of our hospital (H29-300). Before start of PBT, a written informed consent for their treatment was obtained from each patient, but the consent for this retrospective analysis was waived. All patients were followed up for $>3$ years or until death. For the first 2 years after PBT, all patients were followed every 3 months. Thereafter, the follow-up period was

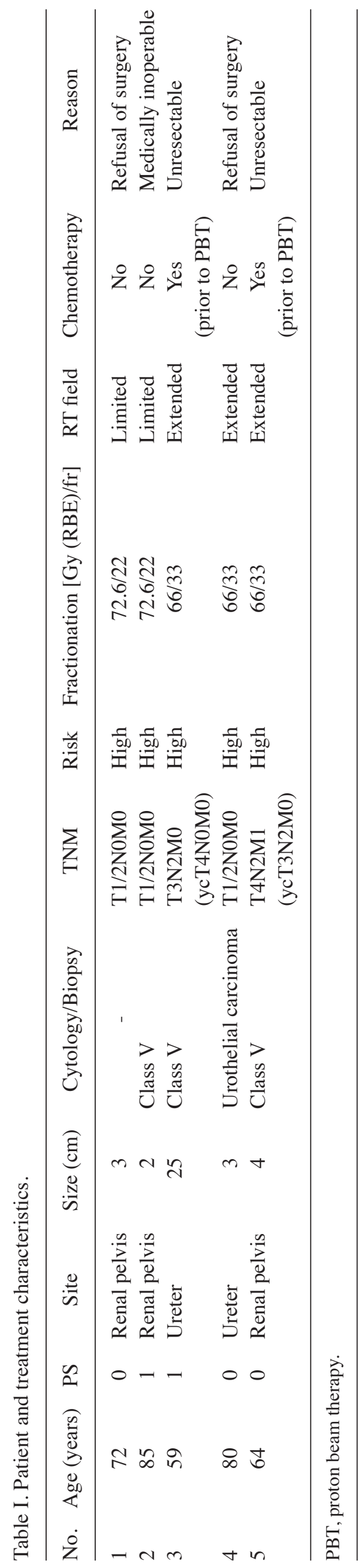


Table II. Summary of treatment outcomes.

\begin{tabular}{rrlclr}
\hline No. & Recurrence & \multicolumn{1}{c}{ Recurrence site } & $\begin{array}{c}\text { Time to } \\
\text { recurrence (months) }\end{array}$ & \multicolumn{1}{c}{ Status } & $\begin{array}{r}\text { Survival } \\
\text { (months) }\end{array}$ \\
\hline 1 & No & & & Alive without disease & 97.5 \\
2 & Yes & Local (within RT field: Primary site) & 36 & Dead with recurrence & 66.3 \\
3 & Yes & Liver & 1 & Dead with recurrence & 4.6 \\
4 & Yes & Local (out of RT field: Bladder) & 48 & Alive with disease & 62.2 \\
5 & Yes & Lung & 28 & Alive with disease & 40.3 \\
\hline
\end{tabular}

Table III. Summary of treatment morbidities.

\begin{tabular}{llll}
\hline & \multicolumn{2}{c}{ Acute } & \\
\cline { 2 - 3 } No. & \multicolumn{1}{c}{ Hematological (grade) } & \multicolumn{1}{c}{ Non-hematological (grade) } & Late (grade) \\
\hline 1 & None (0) & Dermatitis (2) & None \\
2 & None (0) & Dermatitis (1) & Hematuria (2) \\
3 & Anemia (1) & Dermatitis (1), urinary frequency (1) & None \\
4 & Thrombocytopenia (1) & None (0) & GI bleeding (1) \\
5 & Anemia (1), thrombocytopenia (1) & Dermatitis (1), diarrhea (1) & None \\
\hline
\end{tabular}

extended for 3 to 6 months. All patients regularly examined urine cytology, ultrasonography and CT during the follow-up period. The median follow-up time was 51.2 months (range, 4.6-97.5 months). Local recurrences were observed at 36 and 57 months after PBT in 2 patients, but primary tumors were controlled in the other 3 patients. Distant metastases developed in 2 patients. Two patients died of cancer recurrence, and another died due to lung cancer recurrence. The remaining 3 patients are still alive at last follow-up. Thus, 4 patients (80\%) survived for $>3$ years after PBT (Table II).

Table III summarizes treatment-related toxicities according to the National Cancer Institute Common Terminology Criteria for Adverse Effects (CTCAE), version 4.0. With respect to acute toxicity, dermatitis was common but manageable. Non-severe hematological toxicity was observed in all patients who received prophylactic irradiation using extend PBT fields, including 2 patients who received neoadjuvant chemotherapy prior to PBT, and grade 3 myelosuppression in 1 patient who received 6 cycles of neoadjuvant cisplatin and gemcitabine chemotherapy that was not completely resolved before initiation of PBT.

With respect to late toxicities, hematuria and gastrointestinal (GI) bleeding occurred in each case, but no grade $\geq 3$ toxicities were observed.

Case presentation. Case 1: An 80-year-old man (No. 4, Table I), who had a history of surgery for colon cancer and an aneurysm, experienced gross hematuria. Cystoscopic examination revealed hemorrhage from the tumor located at the right ureter, and urine cytology was positive. Retrograde pyelography showed an irregular filling defect of the right proximal ureter and hydronephrosis (Fig. 1A). Computed tomography (CT) revealed a right ureter tumor, and a diagnosis of cT1/2NOM0 right ureter cancer was made (Fig. 1B). Urologists considered radical surgery, but he had a high surgical risk because of his history of abdominal surgery and advanced age. Consequently, he selected nonsurgical treatment and was referred to our hospital to receive PBT. The patient underwent PBT at a total dose of 60/66 Gy (RBE) in 33 fractions over 7 weeks; prophylactic irradiation at a total dose of 36.4/40 Gy (RBE) in 20 fractions using extended PBT fields (Fig. 2A and B) and boost therapy of 23.6/26 Gy (RBE) in 13 fractions was performed with small PBT fields. The scheduled treatment was completed without any severe complications. He developed melena possibly caused by grade 2 small intestinal bleeding at 18 months after PBT, but this was resolved with medication. The primary tumor gradually decreased in size (Fig. 3), but he experienced non-muscle invasive bladder cancer and underwent transurethral tumor resection 48 months after PBT. CT revealed further recurrent tumors in the right ureter at the common iliac level, but not recurrence of the primary lesion, 57 months after PBT. Because he was 86 years old, best supportive care was selected. The patient remains alive at last follow-up and he has enjoyed his daily life for more than 7 months without any treatment.

Case 2: A 72-year-old man (No. 1 in Table I) presented with visible hematuria, and CT revealed a tumor in the left renal pelvis (Fig. 4). He was the first case of PBT for renal pelvis and ureter cancer in our institute. Surgery was proposed, but he refused to receive it. He underwent local PBT at a total dose of 66.0/72.6 Gy (RBE) in 22 fractions (Fig. 5). For initial treatment planning, the clinical target volume (CTV) was defined as the visible tumor plus $10-\mathrm{mm}$ margins in all except caudal direction $(15-\mathrm{mm}$ 
A

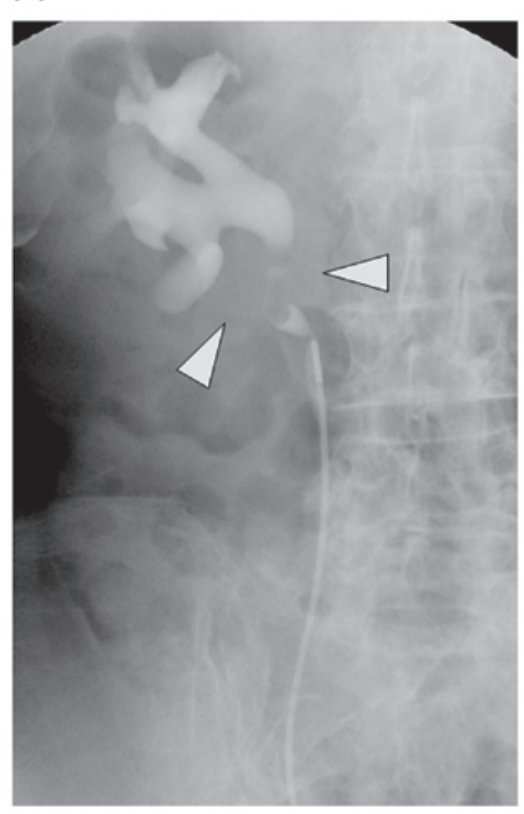

B

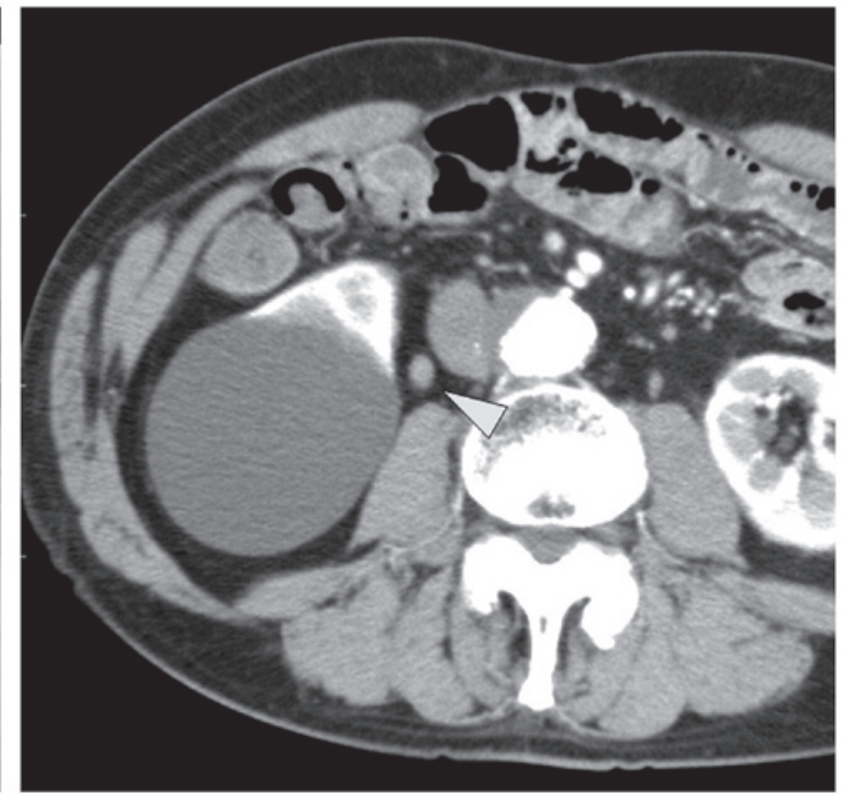

Figure 1. Pyelography and CT prior to treatment (Case 1,80 years old, T2NOM0). (A) An irregular filling defect of the right proximal ureter and hydronephrosis is seen on pyelography, and (B) a visible tumor at the ureter was revealed by CT.

A

B

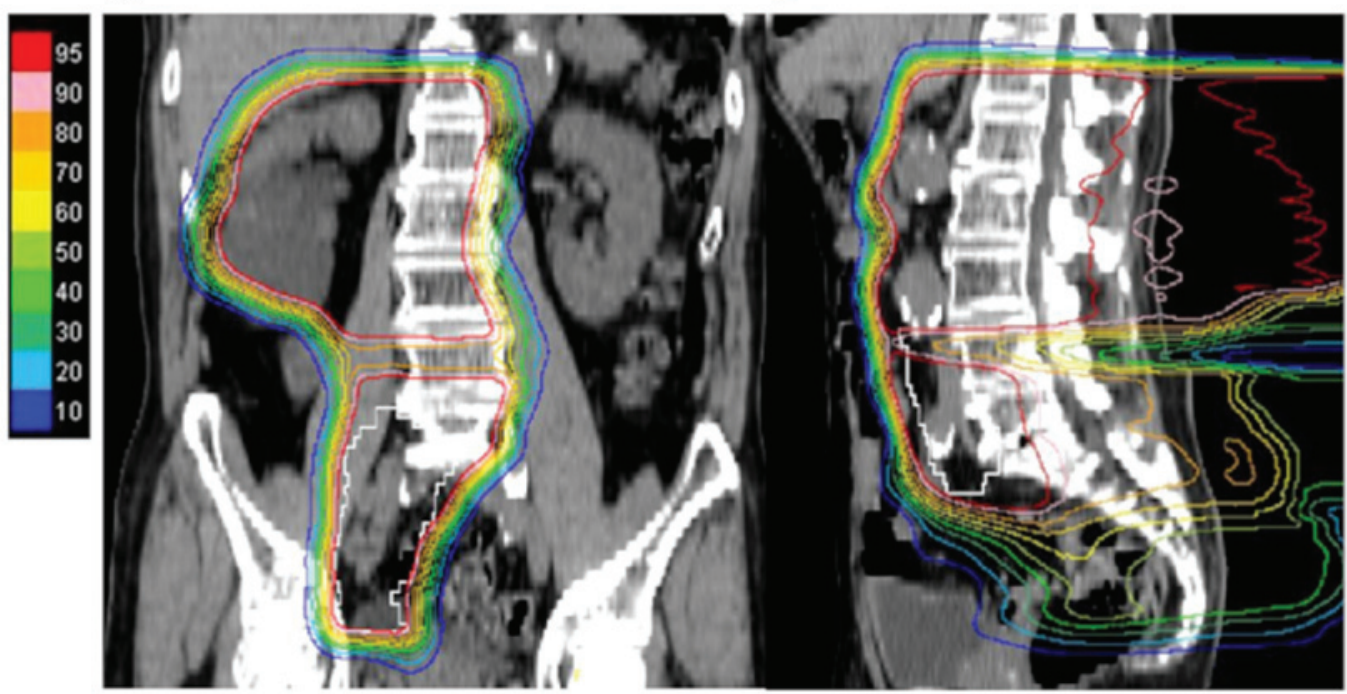

Figure 2. Dose distributions by an extended irradiation field technique using proton beams (Case 1). (A) Coronal and (B) sagittal views.

margin). Although PBT was performed using the respiratory synchronization system, we realized that his left kidney moved approximately $3 \mathrm{~cm}$ in the craniocaudal direction during treatment. After 9.0/9.9 Gy (RBE), the target volume (2nd-CTV) was therefore modified to cover his kidney at the levels of the initial CTV and $>95 \%$ of the prescribed dose completely covered for the 2 nd-CTV in the remaining 19 fractions [57.0/62.7 Gy (RBE)]. Although he developed grade 2 acute dermatitis, treatment was completed on schedule. The tumor progressively decreased in size, and finally disappeared (Fig. 4). The patient remains alive, and the tumor has been well-controlled for 8 years. Although atrophic changes of the irradiated kidney were observed, his renal function has been maintained.

\section{Discussion}

In the present study, we treated 5 patients with renal pelvis or ureter cancer and experienced two important clinical issues. First, all treatments were completed on schedule, and no severe complications were experienced, even though 3 of the 5 patients were treated using extended RT fields and 2 of them received neoadjuvant chemotherapy due to $\mathrm{T} 4$ disease. Second, total PBT doses of 66.0/72.6 Gy (RBE) in 22 fractions (PBT alone; hypofractionation schedule) for T1-2N0 disease $(n=2)$ and 60.0/66.0 Gy (RBE) in 33 fractions with prophylactic lymph node irradiation for advanced disease $(n=3)$ were delivered without concurrent chemotherapy, per our treatment policy. In 2 cases, local recurrence was observed at 36 months 
A

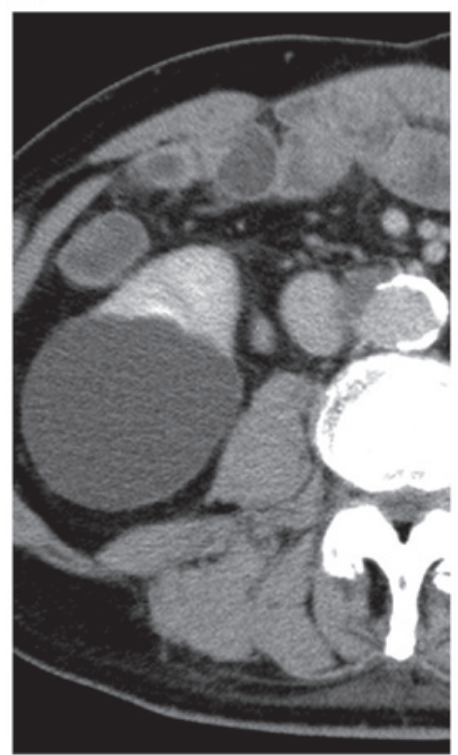

3 months
B

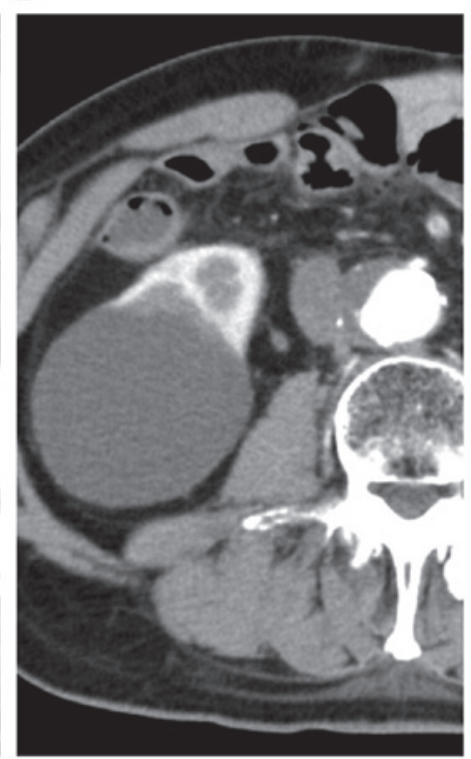

7 months
C

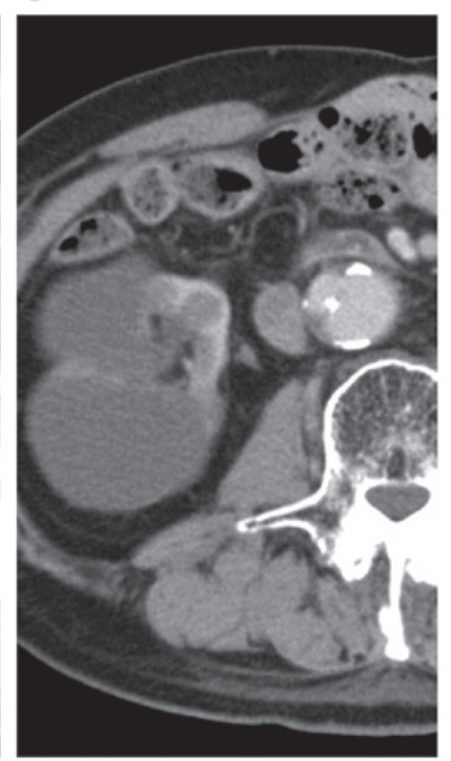

24 months

Figure 3. CT images after proton beam therapy (Case 1). A tumor was gradually shrunken after PBT, and a radiation-induced atrophic change in the right kidney was observed at 24 months after primary treatment; (A) 3 months, (B) 7 months, and (C) 24 months.

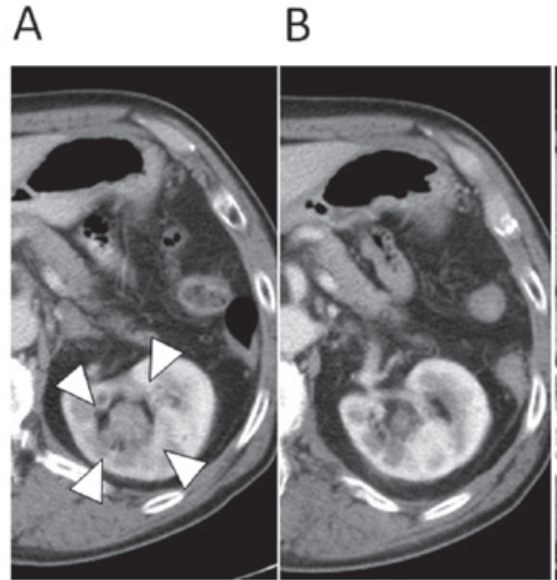

Pre
C

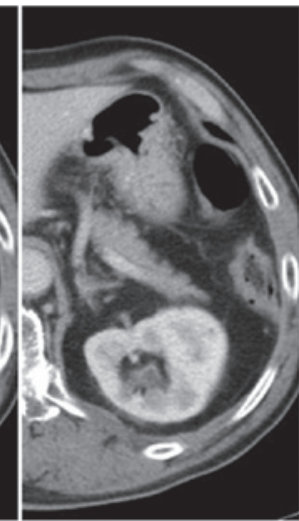

9 months
$\mathrm{D}$

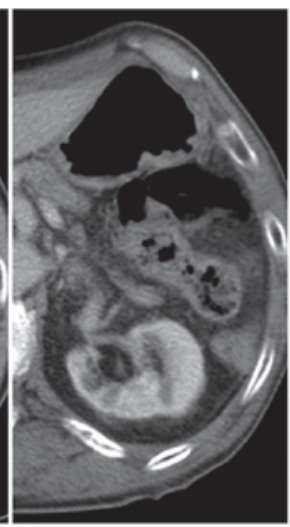

36 months
$\mathrm{E}$

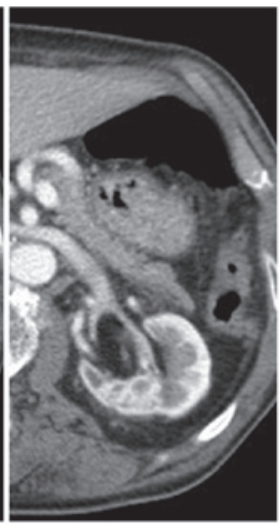

48 months

Figure 4. CT images prior to and following proton beam therapy (Case 2, 72 years old, T2N0M0). A tumor was detected in the left renal pelvis (A) but gradually shrank (B) and was undetectable 9 months after PBT (C). (D) The right half of his kidney irradiated at a high dose was getting smaller and (E) obviously atrophic at 48 months.

(outside the RT field; bladder) and 48 months (primary site) after PBT; however, the tumors of the remaining 3 patients were locally controlled. Consequently, 4 patients $(80 \%)$ survived for $>3$ years, and 1 has experienced no recurrence 97 months after PBT.

Patients with carcinoma of the renal pelvis or ureter are usually treated with surgery. While nephron-sparing approaches are used to treat early-stage disease (3), radical nephroureterectomy, in which Gerota's fascia with the ipsilateral ureter and the bladder cuff are removed, is required for treatment of advanced cases (20). Furthermore, systemic chemotherapy is often provided in both the neoadjuvant and adjuvant settings. In this report, we defined CTV as the primary tumor plus margins for T1-2N0 disease, whereas we used a patch-fields technique to cover large CTV, including the renal pelvis, entire ureter, and regional lymph nodes for 3 cases with advanced disease (Fig. 2). Therefore, the treatment area in this study was similar to that targeted by surgery.

RT for renal pelvis or ureter cancer is usually used with palliative intent or as adjuvant treatment in the postoperative setting. Because locoregional failures after surgery have been observed in $9-15 \%$ of patients with low-grade, low-stage disease but in 30-50\% of patients with high-grade and/or advanced disease $(21,22)$, postoperative RT to eliminate microscopic residual disease appears to significantly reduce local failure risk of advanced disease compared to the surgery alone (5). However, damage to healthy, radiosensitive tissue, such as the GI tract, close to the RT target are concerns in 
A

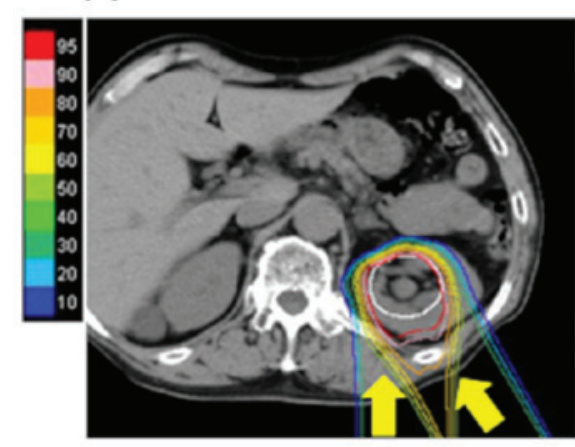

D

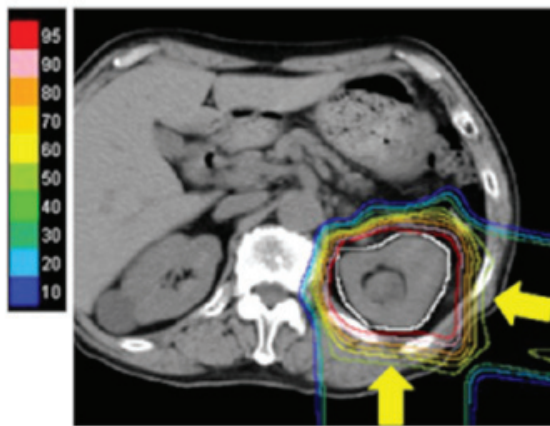

B

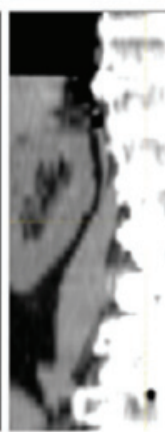

E

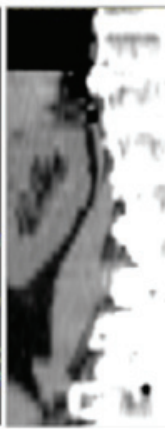

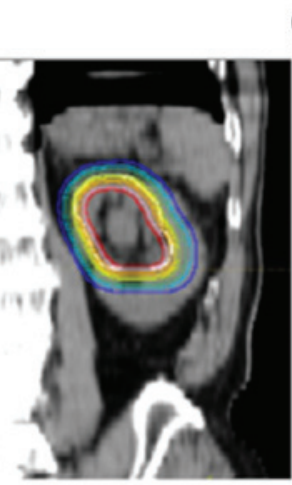

C

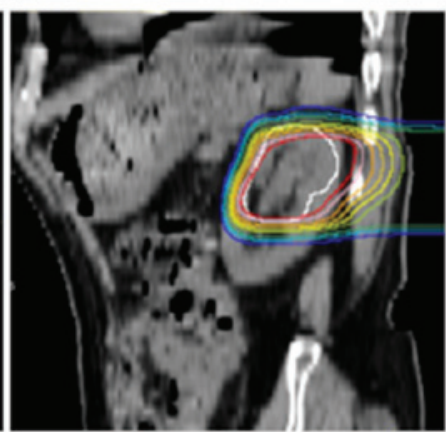

$\mathrm{F}$

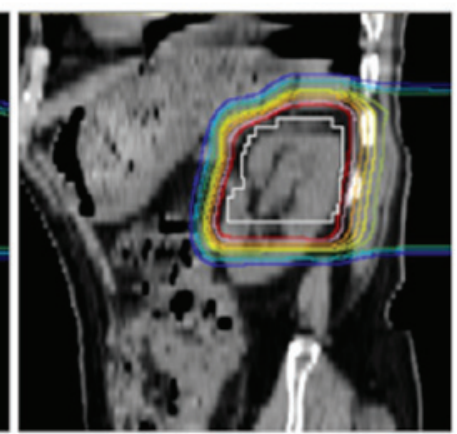

Figure 5. Dose distributions by localized proton beam fields (Case 2). Axial, coronal, and sagittal views for the initial treatment plan (A, B, and C, respectively) and for the modified plan which covered the patient's kidney at the level of initial CTV and $>95 \%$ of the prescribed dose completely covered for the 2 nd-CTV (D, E, and F, respectively). Both treatment plans were performed using same CT images.

the curative treatment setting, which requires high irradiation doses for tumor control. To our knowledge, no guidelines exist for management of inoperable patients with upper urinary tract cancer due to extensive disease burden, a solitary kidney, poor performance status, or patient refusal to undergo surgery. Therefore, new RT approaches that allow sparing of organs at risk from high RT dose areas are required for successful curative treatment of this disease.

PBT is used as highly-conformal RT and possesses the possibility to deliver curative doses not only to the tumor sites but also prophylactically to lymph node areas while avoiding toxic doses to healthy tissues. Protons from posterior beams are directed at the primary site and regional lymph nodes, while they are withheld from the GI tract. Furthermore, the irradiation doses administered to the spinal cord are also acceptable (Fig. 3). As a result, we were able to deliver sufficient irradiation doses to locally control not only small (T1-2) but also advanced (T3-T4) tumors. In the present study, local failure was observed in 2 cases, but 1 recurrence occurred within the prophylactic RT field at 2 years after initial recurrence in the bladder. Furthermore, the local recurrence in the other patient developed 5 years after hypofractionated PBT. Therefore, our treatment strategy appears to improve patient survival compared to chemotherapy alone or best supportive care. Recently, some studies have suggested the utility of stereotactic RT for localized renal pelvis and/or ureter cancer. Maehata et al reported 3 cases of inoperable localized T2N0M0 ureter carcinoma treated with stereotactic body radiotherapy (SBRT). In their study, no acute adverse events were observed, and tumor control was obtained in 2 of the 3 patients (15). When high-dose local irradiation is delivered to T1-2 tumors, local tumor control can be achieved.

Considering the outcomes of a series of renal pelvis and ureter cancer surgeries, treatment of regional lymph nodes appears to be necessary for T3-4 disease, irrespective of the presence of lymph node metastasis (21-23). In the present study, 3 patients who received PBT with prophylactic lymph node irradiation using extended fields did not develop further lymph node metastasis, although 1 patient had T4N2 disease. Therefore, our treatment policy for advanced tumors appears to be reasonable. However, distant metastases in the lung and liver were observed in two patients with T4 disease, despite administration of systematic chemotherapy prior to PBT and prophylactic lymph node irradiation. The utility of adjuvant chemotherapy following PBT should be further evaluated in future prospective studies.

There were several limitations to this case report. This report was retrospective observation and of a limited number of patients. Staging of renal pelvis and urether cancer was in accordance with clinical findings, not with pathological findings. Further studies including a larger number of patients are also needed to validate the effectiveness of definitive PBT for the disease.

In conclusion, this is the first report of curative PBT for localized renal pelvis and ureter cancers. The present results demonstrate that PBT may be effective and feasible as a curative treatment modality for the disease, and it probably has the potential to become a good candidate as an alternative radiotherapy for inoperable patients with early or locally advanced upper urinary tract cancer patients. Prospective 
studies are required to confirm the efficacy of PBT in this setting.

\section{Acknowledgements}

Not applicable.

\section{Funding}

The present study work was supported by JSPS KAKENHI (grant no. JP17K10467).

\section{Availability of data and materials}

The datasets generated during and/or analysed during the present study are not publicly available for maintaining the privacy of the patients but are available from the corresponding author on reasonable request.

\section{Authors' contributions}

TI made substantial contributions to acquisition of data and drafting the manuscript. HI made substantial contributions to conception, design and revising the manuscript. YS, KO, MM, and TN made substantial contributions to acquisition, analysis and interpretation of data. HS also made substantial contributions to interpretation of data, revising the manuscript, and the final approval of the version to be published. All authors read, approved the final manuscript and agreed to be accountable for all aspects of the work in ensuring that questions related to the accuracy or integrity of any part of the work were appropriately investigated and resolved.

\section{Ethics approval and consent to participate}

The present study was approved by the Institutional Review Board of Tsukuba University Hospital (H29-300). For this type of study formal consent was not required. Information about the current study was disclosed to patients instead of obtaining their written informed consent, and patients who declined to participate were excluded.

\section{Patient consent for publication}

Not applicable.

\section{Competing interests}

The authors declare that they have no competing interests.

\section{References}

1. Kirkali $\mathrm{Z}$ and Tuzel E: Transitional cell carcinoma of the ureter and renal pelvis. Crit Rev Oncol Hematol 47: 155-169, 2003.

2. Huben RP, Mounzer AM and Murphy GP: Tumor grade and stage as prognostic variables in upper tract urothelial tumors. Cancer 62: 2016-2020, 1988.

3. Hutchinson R, Haddad A, Saqalowsky A and Margulis V: Upper tract urothelial carcinoma: Special considerations. Clin Adv Hematol Oncol 14: 101-109, 2016.

4. Soderdahl DW, Fabrizio MD, Rahman NU, Jarrett TW and Bagley DH: Endoscopic treatment of upper tract transitional cell carcinoma. Urol Oncol 23: 114-122, 2005.
5. Cozad SC, Smalley SR, Austenfeld M, Noble M, Jennings S and Raymond R: Adjuvant radiotherapy in high stage transitional cell carcinoma of the renal pelvis and ureter. Int J Radiat Oncol Biol Phys 24: 743-745, 1992.

6. Czito B, Zietman A, Kaufman D, Skowronski U and Shipley W: Adjuvant radiotherapy with and without concurrent chemotherapy for locally advanced transitional cell carcinoma of the renal pelvis and ureter. J Urol 172: 1271-1275, 2004.

7. Ding T, Zheng Z, Xu R and Zhou C: Prognostic factors and outcomes of primary transitional cell carcinoma of the ureter: A population-based study. Oncotarget 8: 65983-65996, 2017.

8. DeLaney TF, Trofimov AV, Engelsman $M$ and Suit HD: Advanced-technology radiation therapy in the management of bone and soft tissue sarcomas. Cancer Control 12: 27-35, 2005.

9. Inada T, Hayakawa Y, Tada J, Takada Y and Maruhashi A: Characteristics of proton beams after field shaping at PMRC. Med Biol Eng Comput 31 (Suppl): S44-S48, 1993.

10. Iwata $\mathrm{H}$, Ishikawa $\mathrm{H}$, Takagi $\mathrm{M}$, Okimoto $\mathrm{T}$, Murayama $\mathrm{S}$, Akimoto T, Wada H, Arimura T, Sato Y, Araya M, et al: Long-term outcomes of proton therapy for prostate cancer in Japan: A multi-institutional survey of the Japanese Radiation Oncology Study Group. Cancer Med 7: 677-689, 2018.

11. Ha B, Cho KH, Lee KH, Joung JY, Kim YJ,Lee SU, Kim H, Suh YG, Moon SH, Lim YK, et al: Long-term results of a phase II study of hypofractionated proton therapy for prostate cancer: Moderate versus extreme hypofractionation. Radiat Oncol 14: 4, 2019.

12. Verma V, Simone CB II and Mishra MV: Quality of life and patient-reported outcomes following proton radiation therapy: A systematic review. J Natl Cancer Inst 110: 2018.

13. Takaoka EI, Miyazaki J, Ishikawa H, Kawai K, Kimura T, Ishitsuka R, Kojima T, Kanuma R, Takizawa D, Okumura T, et al: Long-term single-institute experience with trimodal bladder-preserving therapy with proton beam therapy for muscle-invasive bladder cancer. Jpn J Clin Oncol 47: 67-73, 2017.

14. Hata M, Miyanaga N, Tokuuye K, Saida Y, Ohara K, Sugahara S, Kagei K, Igaki H, Hashimoto T, Hattori K, et al: Proton beam therapy for invasive bladder cancer: A prospective study of bladder-preserving therapy with combined radiotherapy and intra-arterial chemotherapy. Int J Radiat Oncol Biol Phys 64: 1371-1379, 2006

15. Maehata Y, Kuriyama K, Aoki S, Araya M, Marino K and Onishi H: Stereotactic body radiotherapy for localized ureter transitional cell carcinoma: Three case reports. Case Rep Urol 2015: 519897, 2015.

16. Evans JD, Hansen CC, Tollefson MK and Hallemeier CL: Stereotactic body radiation therapy for medically inoperable, clinically localized, urothelial carcinoma of the renal pelvis: A case report. Adv Radiat Oncol 3: 57-61, 2017.

17. Oshiro Y, Mizumoto M, Okumura T, Fukuda K, Fukumitsu N, Abei M, Ishikawa H, Takizawa D and Sakurai H: Analysis of repeated proton beam therapy for patients with hepatocellular carcinoma. Radiother Oncol 123: 240-245, 2017.

18. Okonogi N, Hashimoto T, Ishida M, Ohno T, Terunuma T, Okumura T, Sakae T and Sakurai H: Designed-seamless irradiation technique for extended whole mediastinal proton-beam irradiation for esophageal cancer. Radiat Oncol 7: 173, 2012.

19. Ishikawa H, Hashimoto T, Moriwaki T, Hyodo I, Hisakura K, Terashima H, Ohkohchi N, Ohno T, Makishima H, Mizumoto M, et al: Proton beam therapy combined with concurrent chemotherapy for esophageal cancer. Anticancer Res 35: $1757-1762,2015$

20. Rouprêt M, Babjuk M, Compérat E, Zigeuner R, Sylvester RJ, Burger M, Cowan NC, Böhle A, Van Rhijn BW, Kaasinen E, et al: European association of urology guidelines on upper urinary tract urothelial cell carcinoma: 2015 update. Eur Urol 68: 868-879, 2015.

21. Charbit L, Gendroau MC, Mee S and Cukier J: Tumors of the upper urinary tract: 10 years of experience. J Urol 146: 1243-1246, 1991.

22. Miyao N, Masumori N, Takahashi A, Sasai M, Hisataki T, Kitamura H, Satoh M and Tsukamoto T: Lymph nodde metastasis in patients with carcinomas of the renal pelvis and ureter. Eur Urol 33: 180-185, 1998.

23. Yang D, Chen Q, Song X, Wang J, Che X, Zhu Z, Zheng W and Wang L: Effect of lymph node dissection on the outcomes of upper tract urothelial carcinomas: A meta-analysis. Expert Rev Anticancer Ther 14: 667-675, 2014. International (CC BY-NC-ND 4.0) License. 